\title{
Demonstration of the ultrafast nature of laser produced betatron radiation
}

\author{
K. Ta Phuoc, ${ }^{\text {a) }}$ R. Fitour, A. Tafzi, T. Garl, N. Artemiev, R. Shah, F. Albert, \\ D. Boschetto, and A. Rousse \\ Laboratoire d'Optique Appliquée, ENSTA, CNRS UMR7639, Ecole Polytechnique, Chemin de la Hunière, \\ 91761 Palaiseau, France \\ D-E. Kim \\ Laser Science Laboratory, Department of Physics, POSTECH, San 31 HyoJa-Dong, Pohang, \\ Kyungbuk 790-784, Republic of Korea
}

\author{
A. Pukhov and V. Seredov \\ Insitut für Theoretische Physik I, Heinrich-Heine-Universitat, Duesseldorf, 40225 Duesseldorf, Germany \\ I. Kostyukov \\ Insitute for Applied Physics, 603950 Nizhni Novgorod, Russia
}

(Received 22 May 2007; accepted 12 June 2007; published online 1 August 2007)

\begin{abstract}
This Letter aims to demonstrate the ultrafast nature of laser produced betatron radiation and its potential for application experiments. An upper estimate of the betatron x-ray pulse duration has been obtained by performing a time-resolved x-ray diffraction experiment: The ultrafast nonthermal melting of a semiconductor crystal ( $\mathrm{InSb}$ ) has been used to trigger the betatron x-ray beam diffracted from the surface. An x-ray pulse duration of less than 1 ps at full width half-maximum (FWHM) has been measured with a best fit obtained for 100 fs FWHM. () 2007 American Institute of Physics. [DOI: 10.1063/1.2754624]
\end{abstract}

At the frontier between plasmas and accelerators, the betatron $\mathrm{x}$-ray radiation generated in relativistic laser plasma interaction reproduces the principle of a synchrotron in a millimeter scale. In this laser-based x-ray source, a plasma cavity driven by an intense femtosecond laser replaces the conventional electron accelerator and wiggler. ${ }^{1-4}$ This conceptually new mechanism has been recently demonstrated ${ }^{5-7}$ by the observation of the first $\mathrm{keV}$ x-ray beam generated from a laser produced plasma. Following this result, which has provided a novel approach for $\mathrm{x}$-ray generation, most of the betatron source parameters have been experimentally investigated. However, its most essential feature, the pulse duration, remained unmeasured so far. In this Letter, using time resolved $\mathrm{x}$-ray diffraction we demonstrate for the first time the ultrafast nature of laser produced betatron radiation. This feature finalizes the experimental characterization of the source and demonstrates that it can now be used for applications. The betatron source, combining ultrafast duration, collimation, and $\mathrm{keV}$ energies, becomes one of the very few short pulse x-ray sources emerging from both the accelerator and plasma communities. ${ }^{8-11}$

The detailed theory of betatron radiation has been extensively described in several publications. The principle is as follows: an intense laser focused in a plasma of helium generates, by the way of its ponderomotive force, a relativistic plasma wave with a first period consisting in an ion cavity, the so-called bubble. Thanks to the intense electrostatic fields of the cavity, background electrons can be trapped, accelerated to relativistic energies, and wiggled. The relativistic oscillating motion of the whole electron bunch results in the

\footnotetext{
${ }^{a)}$ Author to whom correspondence should be addressed. Electronic mail: kim.taphuoc@ensta.fr
}

emission of the betatron x-ray beam, which simply consists of incoherent charged-particle radiation. The features of the betatron radiation have their origins at different stages of the process: formation of the plasma cavity, trapping and acceleration of electrons, wiggling. They depend on the laser and plasma parameters. In particular, the x-ray pulse duration is globally determined by the length of the relativistic electron bunch.

To numerically investigate the features of the betatron temporal structure, we have used three-dimensional particlein-cell (PIC) simulations. To simulate the x-ray generation, we use the phenomenological approach described in Ref. 3. The characteristic spatial scale of the electron motion, well resolved in the simulation, is the betatron period. In our PIC code, we follow trajectories of each electron and calculate the emission during the interaction. ${ }^{3}$ We suppose that at any given moment of time, the relativistic electron emits, along its momentum direction, a radiation spectrum defined by the universal function $S\left(\omega / \omega_{c}\right)^{3}$ In the simulation, we selected $\mathrm{x}$-ray photons in the energy range $2 \pm 0.2 \mathrm{keV}$. This energy bandwidth was chosen to increase the sampling and to reduce the stochastic noise. Figure 1 represents the temporal profile of the betatron x-ray pulse obtained in the PIC simulation.

We can see that the expected pulse duration is $\sim 25 \mathrm{fs}$ full width at half-maximum (FWHM). A simple estimation for the X-ray pulse duration can be done if we assume that the accelerating structure-bubble or wake field-is propagating with the constant phase velocity $v_{\mathrm{ph}}$ and background electrons are continuously trapped in this structure. Electrons are accelerated on the distance $L_{\text {acc }}$ and emit x rays that, in turn, propagate with the speed of light $c$. Then, the duration of the x-ray flash at the target is $\tau_{X}=L_{\mathrm{acc}}\left(c-v_{\mathrm{ph}}\right) / c^{2}$. 


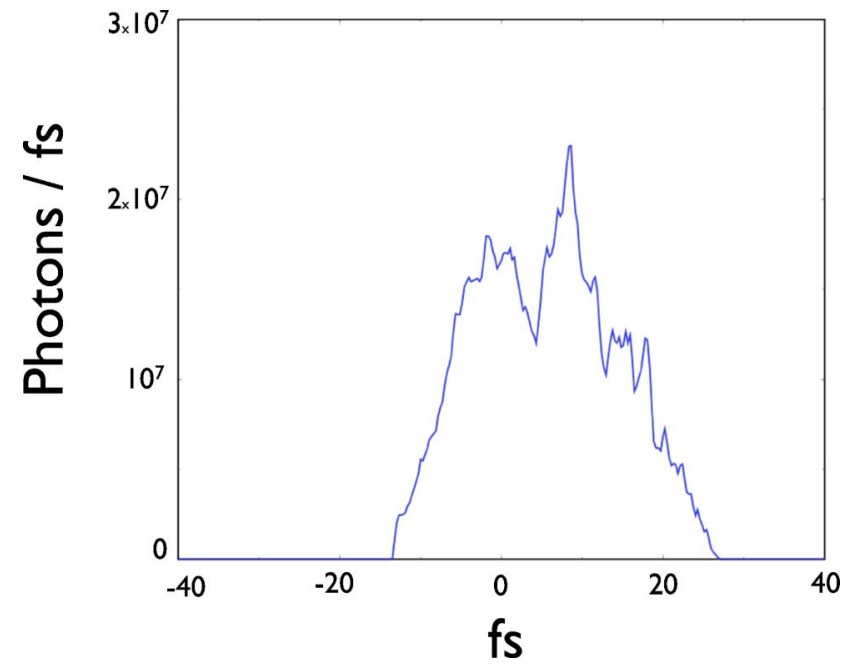

FIG. 1. (Color online) Temporal profile of the betatron x-ray pulse obtained in the PIC simulation. The photon energy is in the bandwidth $2 \pm 0.2 \mathrm{keV}$.

Assuming that the phase velocity of the bubble is defined by the laser group velocity and substituting the linear dispersion relation, we obtain $\left(c-v_{\mathrm{ph}}\right) / c \approx n_{e} / 2 n_{\mathrm{cr}}$ and $\tau_{X} \approx L_{\mathrm{acc}} n_{e} / 2 n_{\mathrm{cr}} c$. Here $n_{\mathrm{cr}}=\left(m c^{2} / e^{2}\right)\left(\pi / \lambda^{2}\right)$ is the critical density for a laser pulse with the wavelength $\lambda$. In practical units, the estimate for the $\mathrm{x}$-flash duration is

$$
\tau_{X}(\mathrm{fs}) \approx 1.5 \times 10^{-18} L_{\mathrm{acc}}(\mathrm{mm}) n_{e}\left(1 / \mathrm{cm}^{3}\right)(\lambda[\mu \mathrm{m}])^{2} .
$$

Substituting the experimental acceleration length $L_{\text {acc }}$ $\approx 3 \mathrm{~mm}$, the laser pulse wavelength $\lambda=800 \mathrm{~nm}$, and the plasma density $n_{e}=10^{19} \mathrm{~cm}^{-3}$, we obtain from (1) for the expected $\mathrm{x}$-flash duration $\tau_{X} \approx 29 \mathrm{fs}$ (full length), which is in agreement with the more detailed PIC analysis.

Experimentally, the measurement of subpicosecond $\mathrm{x}$-ray pulses remains a challenge as no direct technique can provide the required resolution. Indirectly, information can be inferred by measuring the duration of the electron bunch (electro-optic sampling, transition radiation) or by crosscorrelating the visible part of the synchrotron radiation together with a femtosecond laser pulse. ${ }^{11,12}$ To directly probe the $\mathrm{x}$-ray pulse, a cross correlation between the $\mathrm{x}$-ray pulse and an ultrafast Bragg switch, triggered by a visible femtosecond pulse, can be used. ${ }^{13}$ The Bragg switch being capable to absorb or diffract $\mathrm{x}$ rays, the information on the $\mathrm{x}$-ray pulse duration is obtained by recording its intensity $I(\Delta t)$ (after diffraction by the switch) as a function of its delay $\Delta t$ with respect to the visible pulse that triggers the switch. As shown in Fig. 2(a), it corresponds to the product of convolution $I(\Delta t)=\int R(t) I_{X}(t-\Delta t) d t$, where $R(t)$ and $I_{X}(t)$ are, respectively, the temporal profiles of the switch and the X-ray pulse. If the Bragg switch $R(t)$ is known, the x-ray pulse profile $I_{X}(t)$ can be obtained using a simple deconvolution.

A possible Bragg switch uses the phase transition of nonthermal melting, for which a solid crystalline surface is transformed into a disordered state following the excitation with a femtosecond laser. ${ }^{11,14-16}$ It has been characterized in a number of time resolved x-ray diffraction experiments using the laser-plasma $K \alpha$ and the femtosecond synchrotron sources. The shortest transition observed, corresponding to
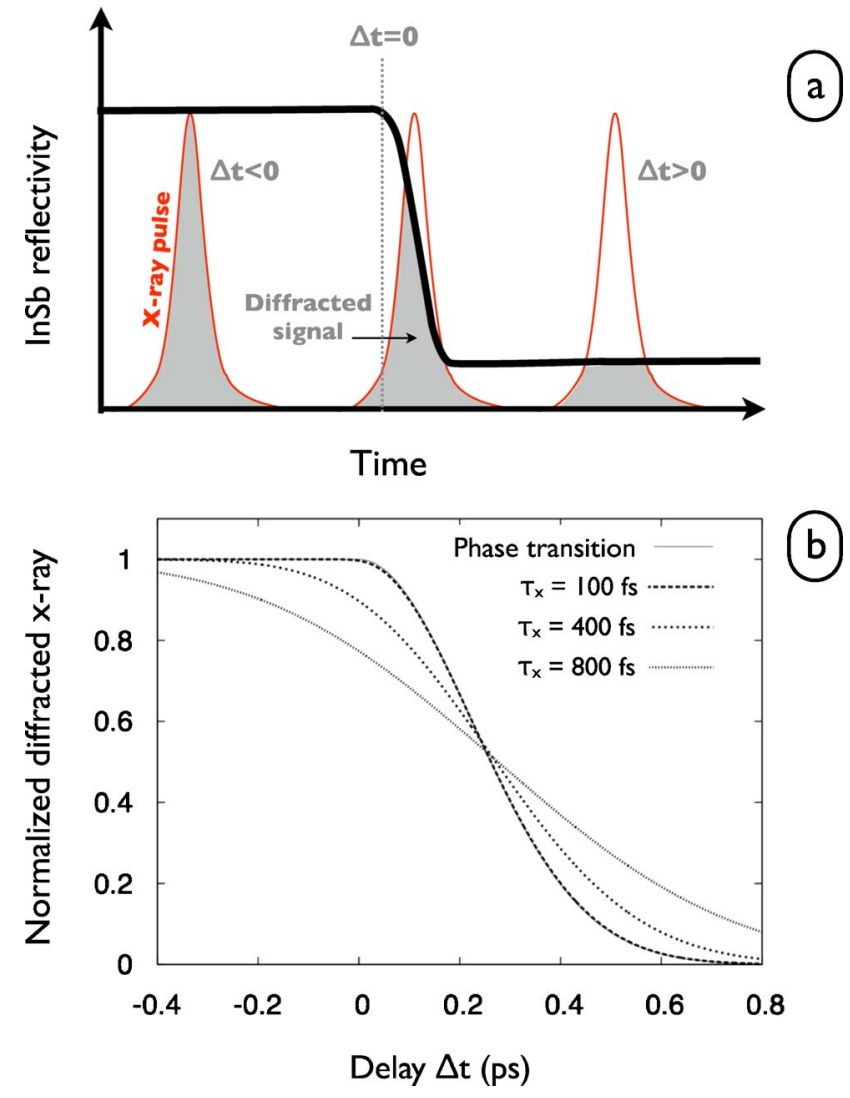

FIG. 2. (Color online) (a) Principle of the x-ray pulse measurement using the nonthermal melting as a Bragg switch. The filled areas represent the signal that can be recorded experimentally. (b) X-ray intensity diffracted by the Bragg switch $I(\Delta t)=\int R(t) I_{X}(t-\Delta t) d t$ for $\tau_{x}=100,400$, and $800 \mathrm{fs}$.

the time for the disorder to appear and the reflectivity to fall, is 200 fs (fall from $90 \%$ to $10 \%$ of the incident $\mathrm{x}$-ray intensity). ${ }^{11}$ This implies that this Bragg switch does not allow the exact determination of $I_{X}(t)$, but can nevertheless provide an upper limit of the pulse duration in the femtosecond time scale. Here we have chosen the solid-liquid phase transition in indium antimonide (InSb) to characterize the betatron pulse duration. We have considered both the InSb reflectivity and the $\mathrm{x}$-ray pulse intensity as Gaussian functions, respectively $R(t)=e^{-\left(1.17 t / T_{p t}\right)^{2} / 2}$ and $I_{X}(t)=e^{-\left(2.35 t / \tau_{X}\right)^{2} / 2}$. Here $T_{p t}$ is the time of the phase transition (at half the amplitude of the reflectivity drop) and $\tau_{X}$ is the FWHM x-ray pulse duration. $T_{p t}$ in InSb has been determined on the basis of the experiments performed with two different x-ray sources: the $K \alpha$ radiation $\left(\tau_{X}=350 \mathrm{fs}\right.$ ) (Ref. 16) and the femtosecond synchrotron beam $\left(\tau_{X}=80 \mathrm{fs}\right) .{ }^{11}$ A duration $T_{p t}=260 \mathrm{fs}$ reproduces the results obtained at these experiments. Assuming this transition time, Fig. 2 shows how $I(\Delta t)$ varies when the transition is probed with $\mathrm{x}$-ray pulses with duration $\tau_{X}=100,400$, and 800 fs. As $\tau_{X}$ increases, the fall time of $I(\Delta t)$ is increased by $\sim 2 \tau_{X}$. We see in Fig. $2(\mathrm{~b})$ that the limit of this method for the measurement of an $\mathrm{x}$-ray pulse is on the order of $100 \mathrm{fs}$ mainly because transitions measured with $\tau_{X} \lesssim 100$ fs are not distinguishable. A faster phase transition time would allow a higher resolution.

The experiment has been performed at the Laboratoire d'Optique Appliquée using a 50 TW, 30 fs titanium-doped 


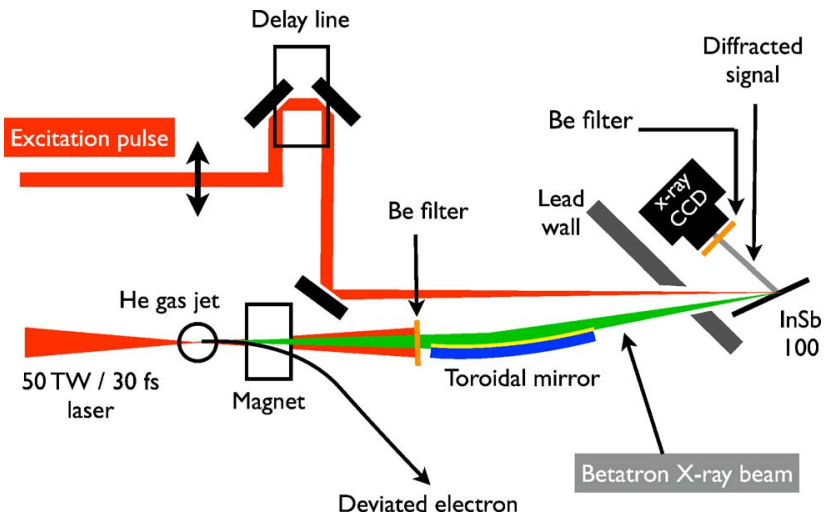

FIG. 3. (Color online) Visible pump, x-ray probe experimental setup. A $30-\mathrm{cm}$-long toroidal mirror is used to collect and focus the $\mathrm{x}$-ray beam onto an asymmetrically cut (100) InSb sample. The phase transition is triggered using a synchronized $30 \mathrm{fs}$ laser pulse at $820 \mathrm{~nm}$.

sapphire (Ti:Sa) laser operating at $10 \mathrm{~Hz}$ in chirped-pulse amplification mode. The experiment setup is displayed in Fig. 3. In this typical pump-probe geometry, two laser beams are involved: the first is used to produce the betatron $\mathrm{x}$-ray beam (probe beam), and the second is used to excite the InSb (pump beam). For the production of betatron radiation, we used the main arm of the laser ( $1 \mathrm{~J}, 30 \mathrm{fs}$ ) focused with an $\mathrm{f} / 18$ off-axis parabolic mirror onto the edge of a supersonic helium gas jet (diameter $3 \mathrm{~mm}$ ). The laser distribution in the focal plane was Gaussian with a waist $w_{0} \sim 18 \mu \mathrm{m}$ and vacuum-focused intensities on the order of $3 \times 10^{18} \mathrm{~W} / \mathrm{cm}^{2}$. The plasma density used was $n_{e}=10^{19} \mathrm{~cm}^{-3}$, which corresponds to the "bubble" regime for our laser parameters. In this regime, electrons are efficiently accelerated and wiggled in the wakefield cavity, and these conditions are the most appropriate for an efficient production of betatron radiation. The betatron $\mathrm{x}$-ray beam produced was then collected using a 30 -cm-long toroidal mirror placed at a grazing incidence of 1 degree, and focused onto an asymmetrically cut (100) InSb sample at an angle of 10 degrees from the surface. The x-ray spot size projected onto the sample surface was $1000 \mu \mathrm{m}$ $\times 150 \mu \mathrm{m}$. For this setup, the throughput of the collectionfocusing system is in the order of $1 \%$. Meanwhile, electrons accelerated were deviated using a permanent magnet, measured in space and energy using a phosphor screen, and the laser light was blocked on axis with a $25 \mu \mathrm{m}$ Be filter. The second arm of the laser, $30 \mathrm{fs}, \sim 200 \mathrm{~mJ} / \mathrm{cm}^{2}$ on the sample, was used to optically excite the InSb. It was focused within a $1700 \mu \mathrm{m} \times 300 \mu \mathrm{m}$ spot onto the InSb sample. Generated by a single laser system, the visible pump and x-ray probe pulses are perfectly synchronized and the pump-probe delay $\Delta t$ was adjusted using an optical delay line. In this geometry, the betatron radiation at $1.8 \mathrm{keV}$ with a spectral bandwidth of $15 \mathrm{eV}$ was diffracted by the (111) planes of the sample (Bragg angle: 66.4 degrees) and recorded on a cooled x-ray camera as a function of the pump-probe delay. The spectral $\mathrm{x}$-ray bandwidth diffracted by the crystal was defined by the angular spread of the focused beam (the acceptance of InSb from the rocking curve is much larger). After each shot, the sample was translated with an accuracy better than $10 \mu \mathrm{m}$.

In addition to the uncertainty due to the determination of

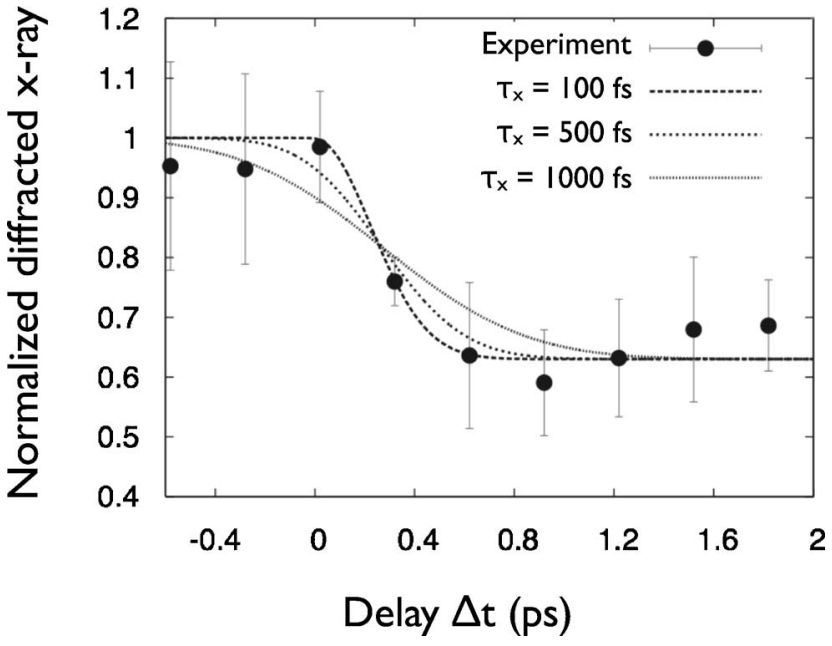

FIG. 4. X-ray intensity diffracted by the excited InSb sample, normalized to the incident $\mathrm{X}$-ray intensity, as a function of the visible pump, $\mathrm{X}$-ray probe delay $\Delta t$. The fits represent $I(\Delta t) / I_{0}$ for $\tau_{x}=100,500$, and $1000 \mathrm{fs}$.

the phase transition time discussed above, the resolution of the measurement was mainly limited by the source fluctuations. Because of the high nonlinearity of the Betatron mechanism, and the fact that the laser intensity was at the threshold for an efficient x-ray generation, the source had significant shot-to-shot fluctuations. Multiple shots for averaged data acquisition and a cross-check of independent experimental results were then essential to prove the reliability of the measurement. Meanwhile, in the geometry of this experiment, the angle between pump and probe beams was 2 degrees. This implies that $\Delta t$ is not constant along the x-ray spot projected onto the sample, and this results in a geometrical $20 \mathrm{fs}$ spread in the observed phase transition time.

Figure 4 displays the measured diffracted x-ray intensity $I(\Delta t)$, normalized by the incident x-ray flux $I_{0}=\int I_{X} d t$, as a function of $\Delta t$. For each time delay, we have recorded 4 to 10 pump-probe shots. The sample was moved between each pump-probe shot, and to account for the possible defects of the sample, we recorded successively three references (probe only) before each pump-probe shot. Each pump-probe shot was then normalized by the mean value $I_{0}$ of the three references. In Fig. 4, the data points correspond to the mean value of the normalized pump-probe shots. The errors bars correspond to the absolute uncertainty on this mean value (standard deviation divided by the square root of the number of shots for each delay). The spread in the data points for $\Delta t<0$ shows the fluctuation of the source [in that case, the excitation is later than the probe and it is straightforward that $I(\Delta t) / I_{0}$ should be unity]. The uncertainty of the mean value of incident $x$-ray intensity is of the order of $\sim \pm 10 \%$.

As expected for a subpicosecond $\mathrm{x}$-ray probe, we observe an ultrafast drop of the diffracted x-ray intensity. The base lines $I(\Delta t) / I_{0}=1 \quad(\Delta t<0)$ and $I(\Delta t) / I_{0}=0.63 \quad(\Delta t$ $>500 \mathrm{fs})$ are clearly defined. The amplitude of the drop is consistent with what is expected for this geometry (the probing depth is deeper than the excitation depth). ${ }^{16}$ The time for the intensity to fall from $90 \%$ to $10 \%$ of the drop amplitude is on the order of $\sim 400 \mathrm{fs}$. A conventional Gaussian fit gives 
a drop time of $290 \pm 30$ fs FWHM. Additional data points in the drop part would, however, be necessary to better define the time delay at which the x-ray signal starts to drop. Here, to retrieve the $\mathrm{x}$-ray pulse duration from the experimental measurement, we have used a direct comparison with the numerically predicted results. We have calculated $I(\Delta t) / I_{0}$ for x-ray pulse durations $\tau_{X}=100,500$, and 1000 fs. The calculated drops are compared to the experimental results in Fig. 4. X-ray pulses with duration $\tau_{X}>1$ ps lie clearly outside the error bars. Within the error bars, the experimental result can be fitted with $\mathrm{x}$-ray pulses duration ranging from $1 \mathrm{ps}$ to less than $100 \mathrm{fs}$. Whereas the $1 \mathrm{ps}$ pulse cannot be ruled out because it fits on the extrema of the error bars, the best fit is obtained for $\tau_{X}=100 \mathrm{fs}$ or less. This value is in agreement with the simple estimate and the PIC simulation of the $\mathrm{x}$-ray pulse duration. Further confidence in the estimation also comes from the reproducibility of this result at several independent runs.

The x-ray pulse duration obtained can also be crosschecked with the measurements of the duration of the electrons bunch performed in similar parameter regimes. Indeed, $\mathrm{x}$-ray and electrons pulses have quasisimilar durations. Indirect measurement of the laser-plasma accelerated electron bunch have been performed using different techniques: Sub100 fs (full width) wakefield electron bunch duration has been estimated by the measurement of terahertz radiation ${ }^{17,18}$ (duration $<50 \mathrm{fs}$ ), electro-optic sampling, or from the characterization of the electron bunch profiles ${ }^{19}$ (duration $<25 \mathrm{fs}$ ) and electron spectrum ${ }^{20}$ (duration $<10 \mathrm{fs}$ ). A betatron $\mathrm{x}$-ray pulse duration on the order of $<100 \mathrm{fs}$ is therefore supported by the duration of electron bunch obtained previously.

In conclusion, the experimental and numerical results demonstrate the expected ultrafast nature of betatron radiation. This finalizes the characterization of the main features of this novel source. The stability and flux will be improved thanks to the development of more intense lasers and/or guiding techniques. The radiation will as well be more collimated and the collection of the entire x-ray beam will be possible. In addition, $\mathrm{x}$-ray optics designed for a larger distance between the source and the application experiment (1200 $\mathrm{mm}$ in this experiment) will make possible a better shielding of the experiment and will significantly improved the signal-to-noise ratio (of the order of 3 in this experiment). The combination of these improvements together with more statistics would allow us to perform a wider range of application experiments. Beyond this pioneering experiment, a technique with a time resolution close to $1 \mathrm{fs}$ is now necessary to measure with high precision the $\mathrm{x}$-ray pulse duration. Whereas $\mathrm{x}$-ray free electron lasers will produce, in the near future, the most powerful femtosecond $\mathrm{x}$-ray source ever, ${ }^{21}$ this complementary emerging source gathers the pool of the very few existing short pulse x-ray sources based on electron acceleration (Thomson scattering, ${ }^{9}$ electron bunch slicing, ${ }^{10}$ or compression ${ }^{11}$ ) and plasma processes. ${ }^{22-24}$ The betatron source can offer remarkable perspectives toward the production of few $10 \mathrm{keV}$ ultrafast $\mathrm{x}$-ray radiation for re- cording atomic movies of transient structures in crystals, amorphous solids, and warm dense plasmas using femtosecond x-ray diffraction and absorption spectroscopy. ${ }^{25,26}$

R.S. is supported by CNRS and the National Science Foundation under Grant No. 0502281. D.-E.K. would like to acknowledge the support of the Korean Scientific Foundation under the NRL program (XXXXX[HAC5]) and the STAR program. T.G. is supported by the European Marie Curie research training network FLASH (Grant No. MRTN-CT2003-503641).

${ }^{1}$ E. Esarey, B. A. Shadwick, P. Catravas, and W. P. Leemans, Phys. Rev. E 65, 056505 (2002).

${ }^{2}$ I. Kosyukov, A. Pukhov, and S. Kieselev, Phys. Plasmas 10, 4818 (2003).

${ }^{3}$ S. Kiselev, A. Pukhov, and I. Kostyukov, Phys. Rev. Lett. 93, 135004 (2004).

${ }^{4}$ W. P. Leemans, E. Esarey, J. van Tilborg, P. A. Michel, C. B. Schroeder, Cs. Toth, C. G. R. Geddes, and B. A. Shadwick, IEEE Trans. Plasma Sci. 30, 8 (2005).

${ }^{5}$ A. Rousse, K. Ta Phuoc, R. Shah, A. Pukhov, E. Lefebvre, V. Malka, S. Kieselev, F. Burgy, J-P. Rousseau, D. Umstadter, and D. Hulin, Phys. Rev. Lett. 93, 135005 (2004).

${ }^{6}$ K. Ta Phuoc, R. Shah, A. Pukhov, V. Malka, S. Kiselev, D. Umstadter, F. Burgy, J. P. Rousseau, and A. Rousse, Phys. Plasmas 12, 023101 (2005).

${ }^{7}$ K. Ta Phuoc, S. Corde, R. Shah, F. Albert, R. Fitour, J-P. Rousseau, F. Burgy, B. Mercier, and A. Rousse, Phys. Rev. Lett. 97, 225002 (2006).

${ }^{8}$ M. M. Murnane, H. C. Kapteyn, M. D. Rosen, and R. W. Falcone, Science 251, 531 (1991).

${ }^{9}$ R. W. Schoenlein, W. P. Leemans, A. H. Chin, P. Volfbeyn, T. E. Glover, P. Balling, M. Zolotorev, K.-J. Kim, S. Chattopadhyay, and C. V. Shank, Science 274, 236 (1996).

${ }^{10}$ R. W. Schoenlein, S. Chattopadhyay, H. H. W. Chong, T. E. Glover, P. A. Heimann, C. V. Shank, A. A. Zholents, and M. S. Zolotorev, Science 287, 2237 (2000).

${ }^{11}$ A. M. Lindenberg, J. Larsson, K. Sokolowski-Tinten et al., Science 308, $392(2005)$

${ }^{12}$ A. L Cavalieri, D. M. Fritz, S. H. Lee et al., Phys. Rev. Lett. 94, 114801 (2005).

${ }^{13}$ P. H. Bucksbaum and R. Merlin, Solid State Commun. 111, 535 (1999).

${ }^{14}$ C. W. Siders, A. Cavalleri, K. Sokolowski-Tinten, Cs. Tth, T. Guo, M. Kammler, M. Horn von Hoegen, K. R. Wilson, D. von der Linde, and C. P. J. Barty, Science 286, 1340 (1999).

${ }^{15}$ K. Sokolovski-Tinten, C. Blome, C. Dietrich, A. Tarasevitch, M. Horn von Hoegen, D. von der Linde, A. Cavalleri, J. Squier, and M. Kammler, Phys. Rev. Lett. 87, 225701 (2001).

${ }^{16}$ A. Rousse, C. Rischel, S. Fourmaux, I. Uschmann, S. Sebban, G. Grillon, P. Balcou, E. Forster, J-P. Geindre, P. Audebert, J-C. Gauthier, and D. Hulin, Nature 410, 65 (2001).

${ }^{17}$ J. van Tilborg, C. B. Schroeder, C. V. Filip, Cs. Toth, C. G. R. Geddes, G. Fubiani, E. Esarey, and W. P. Leemans, Phys. Plasmas 13, 056704 (2006).

${ }^{18}$ J. Faure, Y. Glinec, G. Gallot, and V. Malka, Phys. Plasmas 13, 056706 (2006).

${ }^{19}$ S. P. D. Mangles, A. G. R. Thomas, M. C. Kaluza et al., Phys. Rev. Lett. 96, 215001 (2006).

${ }^{20}$ J. Faure, C. Rechatin, A. Norlin, A. Lifschitz, Y. Glinec, and V. Malka, Nature 444, 737 (2006).

${ }^{21}$ http://www.xfel.net/en/index.html; www-xfel.spring8.or.jp/; http://wwwssrl.slac.stanford.edu/lcls/

${ }^{22}$ A. Rousse, P. Audebert, J. P. Geindre, F. Fallis, J. C. Gauthier, A. Mysyrowicz, G. Grillon, and A. Antonetti, Phys. Rev. E 50, 5393 (1994).

${ }^{23}$ E. Seres, J. Seres, and C. Spielmann, Appl. Phys. Lett. 89, 181919 (2006).

${ }^{24}$ J. Seres, E. Seres, A. J. Verhoef, G. Tempea, C. Streli, P. Wobrauschek, V. Yakovlev, A. Scrinzi, C. Spielmann, and F. Krausz, Nature 433, 596 (2005).

${ }^{25}$ A. H. Zewail, J. Phys. Chem. A 104, 5660 (2000).

${ }^{26}$ R. F. Service, Science 298, 1356 (2002). 